Revue internationale P.M.E.

Économie et gestion de la petite et moyenne entreprise

\title{
Les fournisseurs/sous-traitants japonais : quasi-ateliers ou partenaires de leurs donneurs d'ordres?
}

\section{Yveline Lecler}

Volume 4, numéro 2, 1991

URI : https://id.erudit.org/iderudit/1008066ar

DOI : https://doi.org/10.7202/1008066ar

Aller au sommaire du numéro

Éditeur(s)

Presses de l’Université du Québec

ISSN

0776-5436 (imprimé)

1918-9699 (numérique)

Découvrir la revue

Citer cette note

Lecler, Y. (1991). Les fournisseurs/sous-traitants japonais : quasi-ateliers ou partenaires de leurs donneurs d'ordres? Revue internationale P.M.E., 4(2), 137-162. https://doi.org/10.7202/1008066ar

\section{Résumé de l'article}

Les grandes entreprises japonaises, peu intégrées, ont largement recours à la sous-traitance auprès d'entreprises de plus petite taille, avec lesquelles elles entretiennent des relations durables et très étroites. La structure industrielle classiquement reconnue comme caractérisant le Japon repose sur l'image de la pyramide ; c'est-à-dire sur une forme organisationnelle de réseaux d'approvisionnements/fournitures de type hiérarchie verticale. Au sommet de chaque pyramide, se trouve une très grande entreprise, évoluant à l'échelle mondiale, tandis que la base est formée de nombreuses PMI ou travailleurs à domicile, les niveaux intermédiaires entre ces deux extrêmes étant constitués d'entreprises de plus en plus petites et de plus en plus dépendantes du niveau supérieur... Appartenance à un "groupe», forte dépendance, mais aussi transfert de compétences et assistance sont donc les traits caractéristiques de ce système.

Les tensions commerciales croissantes avec les pays occidentaux, notamment les États-Unis, et surtout la forte flambée du yen en 1985 ont provoqué une accélération de l'internationalisation des entreprises japonaises qui ne peut rester, au moins à terme, sans effets sur les sous-traitants du Japon.

Après une description du système de sous-traitance japonais, l'article analyse des évolutions stratégiques actuelles : la délocalisation à l'étranger des grands donneurs d'ordres et l'importation de pièces étrangères par les entreprises japonaises au Japon. C'est de ses évolutions qu'apparaît, en conclusion, l'esquisse des relations d'approvisionnement de demain.
Ce document est protégé par la loi sur le droit d'auteur. L'utilisation des services d'Érudit (y compris la reproduction) est assujettie à sa politique d'utilisation que vous pouvez consulter en ligne.

https://apropos.erudit.org/fr/usagers/politique-dutilisation/ 
Notes

\title{
Les fournisseurs/sous-traitants japonais : quasi-ateliers ou partenaires de leurs donneurs d'ordres?
}

\author{
Yveline LECLER * \\ Université Lumière Lyon 2
}

\begin{abstract}
RÉSUMÉ
Les grandes entreprises japonaises, peu intégrées, ont largement recours à la sous-traitance auprès d'entreprises de plus petite taille, avec lesquelles elles entretiennent des relations durables et très étroites. La structure industrielle classiquement reconnue comme caractérisant le Japon repose sur l'image de la pyramide; c'est-à-dire sur une forme organisationnelle de réseaux d'approvisionnements/fournitures de type hiérarchie verticale. Au sommet de chaque pyramide, se trouve une très grande entreprise, évoluant à l'échelle mondiale, tandis que la base est formée de nombreuses PMI ou travailleurs à domicile, les niveaux intermédiaires entre ces deux extrêmes étant constitués d'entreprises de plus en plus petites et de plus en plus dépendantes du niveau supérieur... Appartenance à un "groupe», forte dépendance, mais aussi transfert de compétences et assistance sont donc les traits caractéristiques de ce système.
\end{abstract}

Les tensions commerciales croissantes avec les pays occidentaux, notamment les États-Unis, et surtout la forte flambée du yen en 1985 ont provoqué une accélération de l'internationalisation des entreprises japonaises qui ne peut rester, au moins à terme, sans effets sur les sous-traitants du Japon.

* Ingénieure d'études à l'unité de recherche «Économie des changements technologiques », associé au CNRS. Docteure en Sciences Sociales, Yveline Lecler a d'abord été assistante de recherche à l'École des Hautes Études en Sciences Sociales, Centre de Recherches sur le Japon Contemporain. Elle est actuellement ingénieure d'études au laboratoire d'Économie des Changements Technologiques de l'Université Lumière Lyon 2 où elle poursuit ses travaux sur l'économie japonaise, notamment sur les relations interentreprises et les structures productives. Adresse : Maison Rhône-Alpes des Sciences de L'Homme 14, avenue Berthelot - 69363 Lyon Cedex 07 - France, Tél. : 72.72.64.02 - Télécopieur : 72.72.64.72 
Après une description du système de sous-traitance japonais, l'article analyse des évolutions stratégiques actuelles : la délocalisation à l'étranger des grands donneurs d'ordres et l'importation de pièces étrangères par les entreprises japonaises au Japon. C'est de ses évolutions qu'apparaît, en conclusion, l'esquisse des relations d'approvisionnement de demain.

\section{ABSTRACT}

Japanese big enterprises, less integrated, use to sub-contract a large part of production to smaller enterprises, with whom close relations are established since a long time. The usual image of Japanese industrial structure is that of a pyramid; it means, an organizational form of supplying networks in terms of vertical hierarchy. At the top of each pyramid is there a very big enterprise, behaving on the international scene, while the bottom is composed of numerous small and medium sized firms or home workers. The intermediate levels between these two extremes are made of increasing dependant smaller enterprises.

Belonging to a "group ", strong dependance, but also know-how transfer and assistance are typical features of the Japanese system.

The growing commercial tensions with occidental countries, especially with the United States, and above all the explosion of the yen in 1985, are responsible for the acceleration of Japanese enterprises internationalization. This in turn cannot stay, at least for the long term, without effects on Japanese subcontractors in Japan.

After describing the Japanese sub-contracting system, the article lays the emphasis on the actual strategic evolutions: the delocalisation in foreign countries and the importation of pieces and components from foreign countries by big Japanese manufacturers in Japan. The tomorrow manufacturers/suppliers relations will then be outlined on the base of these evolutions.

\section{RESUMEN}

Las grandes empresas japonesas, poco integradas, recurren a menudo a la subcontrata con empresas más pequeñas, con las que mantienen relaciones estrechas y duraderas. La imagen que caracteriza la estructura industrial de Japón es la piramidal, a saber, una forma de organización de las redes de aprovisionamiento en jerarquía vertical. Arriba de cada pirámide se encuentra una gran empresa que actúa en el marco mundial. La base está constituida por muchas PyME o por trabajadores a domicilio. Los niveles intermedios entre estos dos extremos están constituidos por empresas cada vez más pequeñas y dependientes. Las características más importantes de este sistema son, por un lado, la pertenencia a un "grupo" y la fuerte dependencia, por otro lado, la trasmisión de competencias y la complementariedad.

Las tensiones comerciales con los países occidentales, especialmente Estados Unidos, y sobre todo la fuerte subida del yen en 1985 han provocado el aceleramiento de la internacionalización de las empresas japonesas, fenómeno que puede tener importantes consecuencias a largo plazo sobre los subcontratistas locales. 
Tras describir el sistema japonés de subcontrata, el artículo analiza las actuales evoluciones estratégicas: la entrada en el mercado local de los grandes abastecedores extranjeros y la importación de piezas por parte de las empresas locales.

\section{Introduction}

Les grandes entreprises japonaises, peu intégrées, ont largement recours à la sous-traitance auprès d'entreprises de plus petite taille, avec lesquelles elles entretiennent des relations durables et très étroites. En dehors de la sous-traitance de capacité, tout à fait ponctuelle et ne correspondant pas à la norme en matière de stratégie d'approvisionnement, la structure industrielle classiquement reconnue comme caractérisant le Japon repose sur l'image de la pyramide ; c'est-àdire sur une forme organisationnelle des réseaux d'approvisionnements/ fournitures de type hiérarchie verticale. Au sommet de chaque pyramide se trouve une très grande entreprise évoluant à l'échelle mondiale, tandis que la base est formée de nombreuses PMI ou de travailleurs à domicile. Les niveaux intermédiaires entre ces deux extrêmes sont constitués d'entreprises de plus en plus petites et de plus en plus dépendantes du niveau supérieur. L'ensemble des flux, matières, produits, informations et main-d'œuvre circulent dans cet espace où la domination du plus grand sur le plus petit ne va pas à l'encontre d'une certaine réciprocité des avantages. Ceux-ci se réduisent néanmoins au fur et à mesure que l'on descend dans la pyramide, mais ils restent suffisants pour qu'une réelle conscience d'appartenance à un groupe d'intérêt commun traverse l'ensemble. De même que les contraintes sont transférées en cascade tout au long de la chaîne de fourniture/approvisionnement, l'assistance l'est également.

Bien que l'histoire des relations entre grandes entreprises donneuses d'ordres et PMI sous-traitantes soit faite de restructurations successives ayant pour but final d'adapter l'ensemble du système productif aux nouvelles contraintes de l'environnement, tant national qu'international, les principes organisationnels fondamentaux n'ont pas été remis en cause.

Ainsi, au fil du développement de l'économie japonaise, et notamment lors de la période de haute croissance, les PMI sous-traitantes ont-elles été amenées à se moderniser, afin d'être en mesure de tenir leur rôle dans la division nationale du travail. Un retard trop prononcé de ces dernières aurait, en effet, compromis la progression de toute l'industrie.

Le choc pétrolier de 1973, loin de provoquer un arrêt de ce mouvement a, au contraire, donné un coup d'accélérateur aux restructurations, tant il devenait primordial d'adapter l'ensemble industriel à une période de croissance ralentie. L'environnement étant plus fluctuant et la concurrence plus acerbe, les grandes 
entreprises donneuses d'ordres se sont lancées dans une vague de rationalisation de leur système productif rejaillissant en cascade sur les PMI sous-traitantes vivant dans leur sillage. Le changement technique, tant au niveau production qu'au niveau gestion de production, a diffusé rapidement à la fin des années 1970 et au début des années 1980 dans les grandes entreprises puis dans les PMI, entraînant de fait des changements dans la nature des transactions de soustraitance et dans leur modalité de fonctionnement. L'informatisation en réseaux directs interentreprises a débuté dans les niveaux supérieurs de la pyramide et s'étend petit à petit à l'ensemble.

Enfin, les tensions commerciales croissantes avec les pays occidentaux, notamment les États-Unis, et surtout la forte flambée du yen en 1985, ont provoqué une accélération de l'internationalisation des entreprises japonaises qui ne pourra rester, au moins à terme, sans effets sur les sous-traitants du Japon. Est-ce à dire que les évolutions actuelles sont, contrairement à celles du passé, de nature à remettre en cause le fonctionnement du système d'approvisionnement des entreprises japonaises? Un certain nombre d'éléments dépassant la simple adaptation à un phénomène conjoncturel vont dans ce sens. Il n'est cependant pas pour autant possible de considérer qu'il s'agisse là des prémices d'un nouveau système, c'est-à-dire reposant sur des principes organisationnels fondamentaux différents de ceux qui prévalent aujourd'hui. Il est en effet peu plausible, comme le signalent Trevor et Christie (1988:128) que

[...] les grandes entreprises japonaises ayant encouragé une division $d u$ travail basée sur la spécialisation des fournisseurs, et qui leur permette de créer un système de production efficient, abandonnent un système qui a prouvé sa supériorité en matière de compétitivité, pour se tourner vers une alternative peut-être instable, qui leur est peu familiere.

$\grave{A}$ partir des sources statistiques japonaises existantes ${ }^{1}$ (parfois malheureusement anciennes), dont les tendances ont pu être confirmées sur le terrain lors des enquêtes ${ }^{2}$ que nous avons effectuées entre 1978 et 1989, nous

1. L'ensemble des données chiffrées de cet article provient d'enquêtes effectuées, à fréquence déterminée, par le gouvernement japonais (Secrétariat d'État aux PME principalement). Ces enquêtes ne sont pas toujours disponibles pour le public dans leur intégralité. Les livres blancs annuels qui en rendent compte de façon résumée ne reprennent malheureusement pas systématiquement les mêmes aspects d'une fois à l'autre, mettant en fait l'accent sur les problèmes du moment. Ceci rend plus difficiles les comparaisons sur le long terme, et explique pourquoi des chiffres plus récents ne peuvent parfois pas être mentionnés.

2. Des enquêtes ont été effectuées par l'auteure dans des entreprises japonaises en $1978,1981,1983,1984,1985,1987,1989$. Le thème d'investigation, bien que différent d'une fois à l'autre, a toujours été centré sur les PMI et leurs relations avec les grandes entreprises. C'est au total une trentaine d'entreprises (certaines ayant été visitées plusieurs fois) et de nombreux organismes gouvernementaux, professionnels ou syndicaux qui ont été étudiés. 
tenterons de définir ce système dit de sous-traitance, et de décrire sommairement ses principaux traits caractéristiques. Il s'agira ensuite de discuter, en tenant compte des évolutions actuelles, de l'impact de la hausse du yen et de l'internationalisation de l'industrie japonaise, sur le devenir des relations entre donneurs d'ordres et fournisseurs/sous-traitants. L'ensemble des analyses générales portent, sauf indications contraires, sur l'industrie manufacturière prise globalement, tandis que les exemples plus qualitatifs, issus d'études de cas réalisées par l'auteure, sont empruntés à l'industrie automobile, au machinisme agricole ou à l'électronique grand public ; du moins est-ce le secteur d'appartenance du donneur d'ordres principal ${ }^{3}$.

\section{La relation donneur d'ordres/sous-traitants : une forte dépendance}

Ce qui caractérise le système d'approvisionnement des grandes entreprises japonaises est un fort recours à la sous-traitance, entendu au sens large ${ }^{4}$ auprès d'entreprises très dépendantes de leur chef de file, même si elles travaillent pour plusieurs donneurs d'ordres complémentaires.

\subsection{Un recours massif à la sous-traitance}

La principale raison invoquée par les grandes entreprises pour justifier ce fort taux de recours à la sous-traitance est : " que le sous-traitant possède une technologie spécialisée que nous ne possédons pas » $(57,6 \%$ des firmes, Secrétariat d'état aux PME 1986a ${ }^{5}$ ).

Si l'on ajoute que pour $48,2 \%$ des firmes, la sous-traitance permet de se « concentrer sur les tâches qui constituent nos points forts », alors que 30,6\% des firmes croient que "les séries [étant] plus petites, il est plus rationnel de traiter les petits lots », on se rend compte que des critères technico-stratégiques prévalent aujourd'hui. Certes, la première des raisons a toujours été présente,

3. Certaines PMI enquêtées, spécialisées dans la transformation de matières plastiques, dans le découpage/emboutissage ou encore la fonderie fournissaient également, pour une part certes minime du CA, des entreprises appartenant à d'autres secteurs.

4. L'expression système de sous-traitance qui correspondait relativement bien à la nature de la relation entretenue autrefois avec la plupart des fournisseurs dont le niveau technique était bas est aujourd'hui dépassée par l'évolution des entreprises concernées. Elle reste néanmoins en vigueur, mais doit donc être prise dans un sens très large de division du travail au sein d'un réseau. Les entreprises faisant partie de ce réseau sont d'ailleurs appelées « coopérantes » plutôt que sous-traitantes.

5. Enquête sur la division du travail dans l'industrie manufacturière (seizôgyô bungyôkôzô chôsa), Secrétariat d'État aux PME, février 1986 ; cités dans le livre blanc sur les PME (chûshô kigyô hakusho) 1986, p. 70. 
mais elle ne dominait généralement pas par rapport à des motivations, stratégiques elles aussi, mais basées sur le retard des PMI plutôt que sur leurs capacités technologiques.

En effet, des raisons telles que l'utilisation des bas coûts de production des PMI liés à une main-d'œuvre bon marché ou la possibilité de jouer sur l'élasticité des commandes pour s'adapter aux fluctuations conjoncturelles restent des motivations non négligeables, mais elles ne suffisent plus. De même, sous-traiter parce que les capacités de production sont insuffisantes ou pour éviter d'engager trop de capitaux dans des investissements productifs ne sont plus des raisons fréquemment mentionnées.

Une autre raison avancée est intéressante à signaler, car elle surprend un peu (par la quantité de firmes qui y font référence) dans le contexte actuel de restructuration des réseaux : "parce que nous effectuons des transactions depuis longtemps avec des sous-traitants en qui nous avons confiance » $(46,5 \%$ des firmes).

Que cette raison soit citée par un plus grand nombre d'entreprises que par le passé est quelque peu paradoxal, car, parallèlement, on assiste sur certains segments à une reprise en production interne de travaux auparavant sous-traités, ou au développement de nouveaux sous-traitants dans les domaines concernés par l'émergence de nouveaux matériaux ou de nouvelles pièces. En fait, la durée des relations et la confiance mutuelle jouent très certainement un rôle grandissant, mais pour un nombre de plus en plus limité de sous-traitants ${ }^{6}$.

\subsection{Une forte dépendance : fondement du système}

Le taux de dépendance des PMI vis-à-vis de la sous-traitance ${ }^{7}$ est généralement d'autant plus élevé que l'entreprise est petite. Il est également fonction du secteur d'appartenance : très élevé dans l'industrie du matériel de transport et dans l'ensemble de la mécanique, il est assez réduit dans la chimie ou la pétrochimie. Plus de $80 \%$ des PMI sous-traitantes ont un taux de dépendance vis-à-vis de la sous-traitance supérieur à $80 \%$. Ce taux a tendance à augmenter dans le temps dans les secteurs où il est déjà le plus fort, et à légèrement diminuer dans ceux, plus rares, où il est le plus faible: $92,5 \%$ en 1976 à $94,2 \%$ en 1981 dans le textile, $88,4 \%$ à $89,7 \%$ dans le matériel de transport, contre $56,4 \%$ à $53,6 \%$ dans la chimie ${ }^{8}$.

6. Les laissés-pour-compte ne sont pas forcément abandonnés, ils sont plus généralement retrogradés dans la hiérarchie.

7. Somme totale des transactions de sous-traitance/somme totale des ventes $\times 100$.

8. Chiffres issus de l'enquête sur la situation réelle de l'industrie (kôgyô jittai kihon chôsa) et cités dans le livre blanc sur les PME en graphique (zu de miru chûshô kigyô hakusho) 1983. 
Un fait encore plus significatif pour décrire le fonctionnement de la soustraitance au Japon : la dépendance vis-à-vis du donneur d'ordres principal, laquelle est non seulement très élevée, mais s'inscrit aussi dans la durée. Plus de la moitié des PMI sous-traitantes $(59,1 \%)$ disent n'avoir jamais changé de donneurs d'ordres depuis qu'elles ont commencé les transactions de soustraitance. Ce pourcentage est d'autant plus fort que l'entreprise est grande (73\% des firmes sous-traitantes de 100 à 299 salariés) ${ }^{9}$.

Ceci peut paraître paradoxal alors que l'on parle de la volonté ou de la nécessité de diversification de la clientèle pour les sous-traitants. Cette volonté existe bien dans la majorité des entreprises : $56,7 \%$ des sous-traitants de l'industrie mécanique ${ }^{10}$. Mais elle ne doit cependant pas voiler la réalité : $32,1 \%$ des entreprises interrogées (ind. mécanique) n'ont affaire qu'à un seul donneur d'ordres. Et même si 54,6\% des sous-traitants de cette même industrie ont au moins 3 donneurs d'ordres, il ne faut pas pour autant oublier que pour une moyenne de 6,5 donneurs d'ordres par PMI sous-traitante, le taux de dépendance moyen vis-à-vis du donneur d'ordres principal reste élevé : $62,3 \%^{11}$. Il semble donc qu'en fait, il y ait bien volonté de se diversifier en augmentant le nombre de donneurs d'ordres, mais que chacun ne représente qu'une part minime du chiffre d'affaires, tandis que le donneur d'ordres principal reste largement prédominant. C'est ce qui fait dire au Professeur Sato Yoshio (1983), à propos de la réaction des sous-traitants aux contraintes actuelles, qu'il y a passage de "dépendant envers un donneur d'ordres" à "s'appuyant sur un donneur d'ordres ».

Cette forte dépendance à caractère relativement exclusif permet au donneur d'ordres qui se situe au sommet de la structure, de se centrer sur son métier propre, c'est-à-dire sur ce qui est stratégique pour la maîtrise réelle du produit, principalement la conception puis l'assemblage final. Tout ce qui est intermédiaire, y compris le sous-assemblage et même la conception de certaines pièces et organes complets, peut être délégué à d'autres entreprises, sans risque de perte du contrôle de l'ensemble de la chaîne de production. Le système de fourniture/approvisionnement japonais est communément représenté par une pyramide. Si cette représentation est quelque peu caricaturale, elle reste cependant l'image la plus proche de la réalité.

9. Enquête sur la structure de la division du travail manufacturier (seizô bungyô kôzô chôsa), février 1986 ; in livre blanc sur les PME, 1986 (chûshô kigyô hakusho).

10. Industrie mécanique au sens large, c'est-à-dire industrie des biens d'équipement et de consommation durable. Selon une enquête de la Central Bank of Commercial and Industrial Cooperatives (Shôkô chûkin), Nouvel aperçu sur les PMI sous-traitantes (shitauke chûshô kigyô no shin kyokumen), mars 1983 ; document remis et commenté lors d'une visite de l'auteure à cette organisme.

11. Chiffre d'affaires réalisé avec ce donneur d'ordres/chiffre d'affaires total. 
Toutes les firmes qui travaillent directement ou indirectement pour le donneur d'ordres au sommet d'une pyramide ont conscience d'appartenir à un même "groupe ». Ainsi, une entreprise de premier niveau se définira comme étant de «type Toyota » ou de « type Nissan », tout aussi bien qu'une entreprise de troisième niveau qui pourtant n'est pas en relation directe avec Toyota ou avec Nissan selon le cas. Être de «type Toyota» ou de «type X», ou faire partie du " groupe $X$ », ne signifie pas nécessairement que « $X$ » ait une participation majoritaire au capital, ni même la moindre participation du tout. Les termes type ou groupe font seulement état du donneur d'ordres envers lequel se réalise la dépendance finale. En d'autres termes, les sous-traitants dont le donneur d'ordres principal est « $X$ » ou une entreprise dont le donneur d'ordres principal est « $X$ » (et ainsi de suite) savent toujours qui est le chef de file et se considèrent comme membres du " groupe ». Mais en même temps, cette conscience d'appartenance au donneur d'ordres du sommet n'efface pas l'appartenance première au donneur d'ordres avec qui s'établit la relation directe.

Ainsi, l'image de la pyramide est trop simpliste, la structure étant en fait une imbrication de pyramides, formant elle même une nouvelle pyramide à plusieurs têtes ( $c f$. schéma ci-après).

Le mouvement de diversification des clients qui a lieu au Japon depuis la forte flambée du yen de la fin des années 1980 et l'accélération de la délocalisation à l'étranger qui s'en est suivie ne remettent pas en cause, pour l'instant, ce principe, même si quelques très grands fournisseurs ont pu s'émanciper et devenir tout à fait comparables à leurs homologues occidentaux.

La dépendance dont nous faisons état ne doit pas être perçue comme un facteur purement négatif pour les entreprises sous-traitantes. Certes, les grands donneurs d'ordres sont en position de domination, mais le système leur impose des contraintes qui garantissent l'intérêt mutuel. De fait, les PMI sous-traitantes ont largement bénéficié du système pour se développer et accroître leurs capacités productives.

Le système garantit d'abord au sous-traitant une stabilité des commandes sur du moyen ou long terme, ce qui permet la réalisation des investissements nécessaires. Ainsi, les sous-traitants adaptent petit à petit leurs processus de production aux besoins et spécifications du donneur d'ordres, devenant pratiquement des ateliers séparés de celui-ci. Ceci ne se limite pas aux équipements, mais s'étend aux procédures organisationnelles telles que le «kanban » ou tout autre système de livraisons rapides. Mais pour entretenir un tel degré de dépendance, les donneurs d'ordres doivent également transférer le savoir-faire techniques et les gestionnaires, vers leurs sous-traitants afin de rendre possible une production synchronisée. 


\section{Représentation schématique du système de sous-traitance japonais}

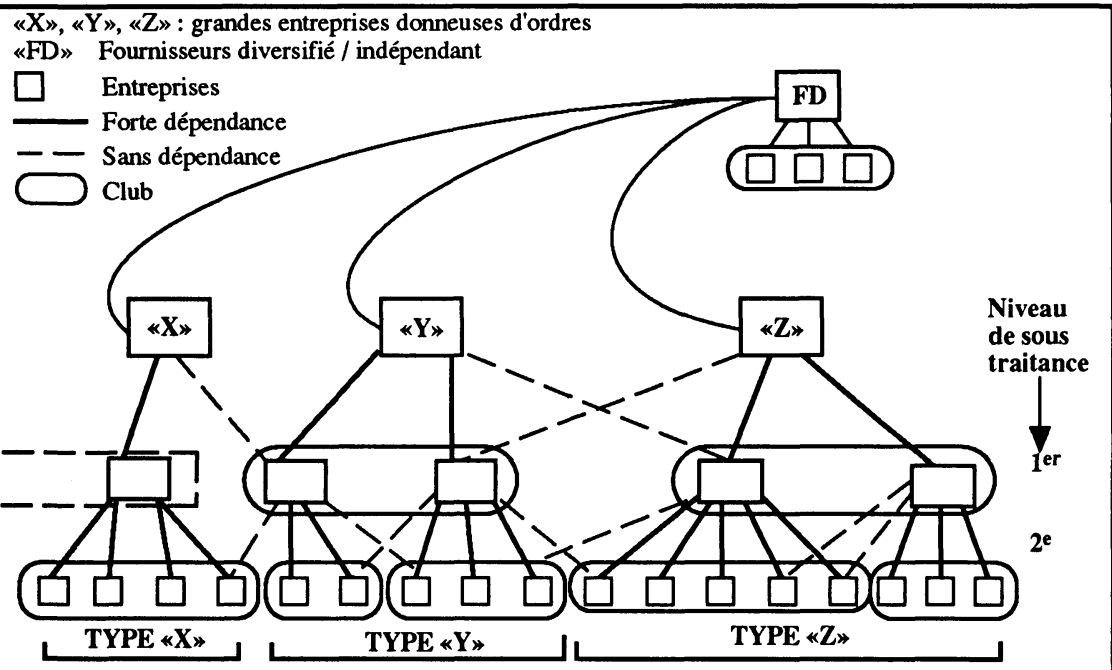

Or, c'est parce que les sous-traitants sont quasiment des ateliers séparés du donneur d'ordres que celui-ci peut leur faire bénéficier de son savoir-faire, soit régulièrement, soit plus ponctuellement lors de l'introduction de nouvelles contraintes, qu'elles soient techniques (lancement d'une nouvelle installation ou de nouveaux produits) ou organisationnelles (flux tendus, synchronisation). C'est également grâce à cette forte intégration que le donneur d'ordres peut faire part à son fournisseur de ses projets d'innovation, sans inquiétude par rapport à une utilisation concurrentielle.

Ce cercle interactif entre les deux partenaires a pour corollaire un intérêt mutuel à ce que la relation reste durable. En effet, le sous-traitant est si intégré à son donneur d'ordres, c'est-à-dire que son dispositif productif est si spécifiquement destiné à répondre aux besoins de celui-ci, qu'il ne peut guère envisager de reconversion totale. Le donneur d'ordres a investi des sommes importantes pour s'assurer les services d'une entreprise dont la culture technique, organisationnelle et humaine est calquée sur la sienne. Bien que l'investissement immatériel relatif à l'entretien des relations avec les fournisseurs/sous-traitants ne soit pas comptabilisé en tant que tel, nous partons du principe que le fonctionnement des clubs, et surtout la masse salariale que représente le personnel affecté aux tâches de conseil/assistance constituent un coût réel qui ne peut être amorti que si la relation est durable. Le transfert de savoir-faire dont il va être question fonctionne en quelque sorte comme une garantie de non-abandon mutuel, bien mieux qu'un contrat. 


\section{L'appui du donneur d'ordres : des formes multiples d'assistance}

La forte dépendance vis-à-vis du donneur d'ordres principal a donc pour contrepartie une assistance qui se manifeste aussi bien sur le plan financier que sur le plan formation ou conseil. Que les mécanismes soient plus ou moins structurés selon que l'on se situe au premier rang ou au troisième, que le contenu soit plus ou moins novateur selon ce même principe ne change rien à la logique qui prévaut.

L'assistance est surtout, et a toujours été, orientée vers l'élévation de la maîtrise technologique des fournisseurs/sous-traitants. On décèle toutefois dans le temps un certain nombre d'évolutions liées aux préoccupations du moment. Une comparaison point par point n'est malheureusement pas possible, car les enquêtes d'une année sur l'autre ne reprennent pas exactement la même formulation. Quoi qu'il en soit, on remarque que sur la période 1973-1979, une grande partie des donneurs d'ordres $(81,1 \%)$ prêtaient ou louaient des machines et équipements à leurs sous-traitants qui n'avaient pas l'assise financière suffisante pour les acquérir par leurs soins. En 1986, bien que ce type d'assistance reste important, il n'est plus le fait que de $69,9 \%$ des donneurs d'ordres. La tendance est la même en ce qui concerne l'attribution de subventions au soustraitant ; formule utilisée par 59,7\% des donneurs d'ordres en 1973-1979 et seulement par $30,7 \%$ en 1986 . La prise de participation au capital, avec $24,1 \%$ (1986) est en constante progression dans le temps.

En ce qui concerne l'assistance/conseil, elle portait principalement sur la gestion des processus de production $(80,8 \%)$ sur la première période considérée, alors qu'elle porte aujourd'hui principalement sur la gestion de la qualité $(79,2 \%)$. L'assistance en matière de formation d'ouvriers, de formation à la gestion des affaires ou à la gestion du personnel semble avoir totalement disparu tandis qu'apparaît une assistance/conseil sur les méthodes de production des nouvelles pièces $(48,3 \%)$, sur l'amélioration des plans et dessins des processus de production $(33,6 \%)$, sur l'amélioration des équipements introduits et de leur utilisation $(30,5 \%)$, sur l'offre de nouvelles technologies d'information, et enfin, bien qu'encore peu fréquent, mais toutefois significatif pour l'avenir, sur la recherche et développement de nouveaux produits ${ }^{12}$.

12. Les chiffres qui représentent des pourcentages de donneurs d'ordres sont issus de l'enquête sur la situation réelle de la sous-traitance (shitauke jittai chôsa) 1979, et de l'enquête sur la situation réelle de l'industrie (kôgyô jittai kihon chôsa) qui a pris la suite. Citées dans les livres blancs sur les PME (chûshô kigyô hakusho) de 1980 et 1986. 
Mais quels sont les mécanismes concrets utilisés pour cette assistance conseil ? Plus ou moins formels selon le niveau, notamment dans la hiérarchie, ils n'ont pas toujours pour vocation première le transfert de compétences. Ils y participent néanmoins soit par l'évaluation des besoins qu'ils permettent, soit plus directement par la diffusion d'information/formation qui les accompagne.

\subsection{Les clubs ou associations de fournisseurs}

Un club ou association de fournisseurs au Japon est une structure permanente et formalisée qui regroupe l'ensemble des entreprises en relation directe avec un donneur d'ordres. Les modalités de sélection des membres sont fonction des entreprises ; mais généralement, un fournisseur, quels que soient sa taille et son domaine d'activité, fait partie du club à partir du moment où il travaille de façon régulière pour un client dont il représente une part significative des besoins pour un type de pièce ou une activité déterminée. Moyennant un droit d'entrée et une cotisation annuelle ${ }^{13}$, il bénéficie de toutes les structures du club. Il est également censé faire bénéficier les autres membres de ses propres avancées, qu'il s'agisse du client chef de file ou d'autres fournisseurs.

Bien que le fonctionnement des clubs diffère d'une entreprise à l'autre, des réunions générales ou en sous-groupes, à périodicité régulière, ont, dans la plupart des cas, pour objectif de diffuser l'information, qu'elle soit technique, commerciale ou gestionnaire, au sein de l'ensemble du « groupe » considéré.

Le niveau général regroupe toutes les entreprises membres, mais ne se réunit qu'une à deux fois par an. C'est un niveau informationnel du donneur d'ordres vers les fournisseurs/sous-traitants qui concerne essentiellement les perspectives de développement de l'année et les problèmes potentiels, notamment en liaison avec l'économie mondiale.

Les réunions en sous-groupes rassemblent un nombre plus limité de firmes, mais avec une fréquence plus soutenue (une fois par mois environ). $\mathrm{Ce}$ niveau spécialisé par domaine d'activité, lequel touche à des aspects beaucoup plus variés, favorise l'échange de compétences entre le donneur d'ordres et les fournisseurs/sous-traitants. En effet, chaque firme membre est appelée (une fois par an environ) à présenter les améliorations qu'elle a entreprises, les problèmes rencontrés et les solutions retenues. Cette présentation peut avoir lieu au tableau noir, ou parfois dans les ateliers même de l'entreprise en question.

Les grands fournisseurs ont eux-mêmes un «club» dont le fonctionnement est calqué sur celui du donneur d'ordres qui se trouve au sommet de la

13. Trente millions de Yens chez Mitsubishi Motors Corporation, par exemple: De Banville et Chanaron 1990. 
pyramide. Les connaissances acquises sont ainsi retransmises en cascade. Cette structure de « club » renforce la synergie entre les acteurs et constitue un important vecteur de transmission des compétences.

En plus de ces actions par groupes, et toujours dans le cadre des activités du « club », il existe également des relations bilatérales, c'est-à-dire du " conseil ${ }^{14}$ » par entreprise, touchant un nombre plus restreint de firmes. C'est notamment le cas de la $\mathrm{R}$ et $\mathrm{D}$ qui, pour des raisons de stratégies et de secret, est rarement l'objet des réunions de groupe.

\subsection{Le personnel chargé de l'assistance/conseil}

Une estimation quantitative de l'importance des actions d'assistance des donneurs d'ordres japonais vers leurs fournisseurs/sous-traitants est totalement impossible, tant elles relèvent de l'informel et passent par un va et vient incessant. Pourtant, elles ne se décident pas au hasard, de façon anarchique, mais s'inscrivent dans une sorte de cadre préétabli qui, lui, est formalisé. En effet, il existe des équipes de conseillers, ou du moins des hommes-conseillers dans les différents services ; il existe également des documents et procédures de contrôle précis à échéance déterminée débouchant sur une évaluation des besoins en compétences nouvelles et sur un éventuel transfert de ces compétences.

\section{a) Les spécialistes de l'assistance/conseil}

Les donneurs d'ordres, selon leur position dans la structure, spécialisent un nombre plus ou moins élevé de salariés dans ces actions d'assistance/conseil. L'équipe de "conseillers ", dont la fonction est de visiter les fournisseurs, de les contrôler et de les assister lorsqu'ils ont des problèmes, est le plus souvent rattachée au service achat/approvisionnement. En plus d'une telle équipe, ou parfois en son absence, les divisions de production ou les divisions techniques ont quelques (4 à 5 en général) « conseillers » qui interviennent chez les fournisseurs/sous-traitants en fonction des besoins.

Une telle organisation des ressources humaines n'est pas l'apanage des seules grandes firmes. Prenons le cas d'une PMI de 245 salariés, sous-traitante de deuxième niveau, qui dispose d'une équipe d'ingénieurs industriels d'une trentaine de personnes. Cette équipe, spécialisée dans le conseil/formation est chargée de couvrir les besoins tant internes qu'externes. Bien que six personnes parmi ces trente soient plus spécialement affectées à l'assistance aux fournisseurs/sous-traitants, toutes peuvent être amenées ponctuellement à travailler avec

14. Le mot « conseil » est utilisé pour traduire les termes japonais de shido, ou anglais de guidance qui n'ont pas d'équivalent strict en français. 
eux. Cet exemple, qui ne constitue pas un cas d'exception, illustre bien, de notre point de vue, la notion de prolongement entre les services du donneur d'ordres et les ateliers séparés que sont les sous-traitants.

\section{b) Les intervenants au quotidien: une réponse aux besoins spécifiques}

En dehors de l'action des " conseillers » (courte durée) et des prêts de personnels dont il va être question (longue durée), il arrive que d'autres salariés aillent chez les fournisseurs pour répondre à un besoin ponctuel d'assistance ou encore que des salariés du fournisseur aillent apprendre quelque chose chez le donneur d'ordres. Ceci est notamment très fréquent lors du lancement d'un nouveau produit ou de la mise en 'euvre d'un nouveau processus technique ou organisationnel (kanban, CAO).

Lors de la mise en place de procédures kanban, ou de l'analyse de la valeur, ou encore de la $\mathrm{CAO}$, une formation est assez systématiquement dispensée par le donneur d'ordres. Elle commence souvent par une sorte de séminaire pour le personnel d'encadrement, afin de lui exposer la démarche. Un stage a ensuite lieu chez le donneur d'ordres pour quelques salariés et enfin, lors de la mise en pratique, une assistance est fournie par la présence fréquente des conseillers chez le sous-traitant. Cette fréquence est très aléatoire selon le problème posé : deux jours au démarrage puis intervention sur demande ; un jour par semaine pendant plusieurs mois pour suivre l'évolution et aider à l'optimisation de la procédure. Il est clair, qu'à moins de multiplier à l'infini les exemples, il soit impossible d'aller plus avant sur ce point.

\subsection{Les " prêts de personnel » et leur effet formateur}

Le «shukko » ou prêt de personnel est une pratique largement utilisée par les entreprises japonaises. Elle relève parfois davantage de l'ajustement des volumes d'emploi que de la volonté d'assister le fournisseur. Les personnes déplacées sont alors des salariés âgés qui pourraient éventuellement retourner chez le donneur d'ordres, mais qui finissent le plus souvent leur vie active là où ils ont été envoyés.

Mais, en plus des « shukko » de ce type, on recourt également à cette pratique pour aider le fournisseur/sous-traitant, soit à résoudre un problème particulier lié par exemple à l'incapacité de recruter un salarié ayant les compétences requises, soit à des fins de formation sur le tas de longue durée. Dans ce cas, les "shukko » sont plutôt (mais pas exclusivement) des jeunes (30 à 35 ans) qui retournent après un ou deux ans chez le donneur d'ordres d'origine. 


\subsection{Le contrôle annuel de la qualité des processus}

Le contrôle annuel de la qualité des processus s'étend à l'ensemble des fonctions de l'entreprise, permettant un diagnostic assez précis des capacités réelles du collectif. Il s'apparente, en plus approfondi, aux " audits qualité » qui se mettent en place actuellement en France dans le cadre du partenariat client/fournisseurs.

Une fois par an, le fournisseur est tenu de remplir un dossier très détaillé où figurent de nombreux indicateurs. Là encore, une formation préalable lui est fournie. Après analyse des indicateurs, les conseillers spécialisés du service d'achats se rendent chez le fournisseur, vérifient la véracité des indications recueillies et attribuent une note pour chaque rubrique. Une appréciation globale A, B, C, parfois D, découle ensuite de cet ensemble. Les entreprises classées A sont considérées comme n'ayant pas besoin d'assistance particulière, du moins tant qu'il n'y a pas lieu d'intégrer de nouvelles contraintes techniques ou organisationnelles. Les entreprises classées $B$ bénéficient par contre d'une aide ponctuelle ou plus durable selon les cas. Parfois elles se voient conseiller diverses formations dispensées par des organismes extérieurs susceptibles de combler les lacunes décelées. Les entreprises classées $\mathrm{C}$ et a fortiori $\mathrm{D}$ ont des inquiétudes à avoir. Si c'est la première fois que cela leur arrive, elles reçoivent immédiatement toute l'assistance nécessaire pour améliorer leur niveau. $\mathrm{Si}$ malgré cela elles ne parviennent pas à atteindre le niveau B au contrôle suivant, elles sont abandonnées ou éventuellement rétrogradées dans la hiérarchie.

Ce contrôle annuel est doublé d'un contrôle exceptionnel lorsqu'un fournisseur réalise une amélioration majeure, afin de vérifier si la dite amélioration entrâne des problèmes sur d'autres segments de la production.

\subsection{La négociation des prix : une occasion de déceler les besoins d'assistance}

Tous les éléments de la relation client/fournisseurs débouchent, au Japon, sur un éventuel transfert de compétences. Prenons l'exemple de la négociation des prix entre client et fournisseurs/sous-traitants pour illustrer notre propos ${ }^{15}$.

En principe, le donneur d'ordres demande au sous-traitant de proposer son prix. Au vu de cette proposition, il indique le prix « correct» selon lui. La négociation s'engage sur ces bases et si le sous-traitant est fortement au-dessus, il doit expliquer comment et pourquoi il arrive au prix proposé. Les points

15. Pour une analyse des principes de détermination des prix de sous-traitance, voir Ueda, Anasz et Yamamoto, 1987. 
faibles sont alors analysés et le donneur d'ordres conseille le sous-traitant pour l'aider à atteindre le prix «correct ». S'il semble incapable de l'atteindre un jour, un autre sous-traitant est sollicité. S'il semble par contre capable d'y parvenir, une période transitoire est accordée pendant laquelle le prix peut-être plus élevé que le prix « correct ».

Lors de la préparation d'un nouveau produit, le donneur d'ordres vient expliquer au sous-traitant ce qu'il veut, quels équipements risquent, de son point de vue, d'être nécessaires. Il exprime également son avis sur le type de moule qu'il faudra prévoir, et le prix auquel il pense souhaitable d'arriver. La discussion s'engage sur ces bases, mais la décision ne peut intervenir qu'après un processus plus ou moins long, selon le degré de complexité ou de nouveauté du produit en préparation. Pendant toute la durée de ce processus de négociation, divers salariés du donneur d'ordres se rendent fréquemment chez le sous-traitant pour voir où en est le dossier et aider, au besoin, à traiter les problèmes qui émergent. Dans certains cas, cela peut donner lieu à l'envoi de quelques personnes chez le donneur d'ordres pour leur apprendre le fonctionnement d'un équipement qui sera nécessaire ou, par exemple, pour montrer aux concepteurs du moule le processus amont et aval par rapport au produit dont leur entreprise aura la charge. Dans d'autres cas, les conseillers de production vont chez le sous-traitant pour une durée de deux semaines à un mois selon les cas, c'est-àdire de la phase de préparation jusqu'au lancement proprement dit.

La stratégie est toutefois différente lorsqu'il s'agit de produits finis qui ont été auparavant fabriqués en interne, quand ils étaient nouveaux et donc relativement stratégiques. Les salariés du sous-traitant pressentis vont alors se former chez le donneur d'ordres avant l'arrêt de la production à l'interne et donc le lancement chez le sous-traitant. La durée de la négociation sur le prix est alors réduite.

Il ressort de tout cela qu'il existe effectivement de nombreuses actions menées par le donneur d'ordres pour aider ses fournisseurs/sous-traitants à élever leur maitrise technologique et organisationnelle. Ces actions de transfert de compétences passent par une intense circulation des hommes et des informations. Toutefois, il ne faut pas mythifier ces pratiques qui représentent parfois des contraintes lourdes pour les fournisseurs et certainement, bien que sous un angle différent, pour le donneur d'ordres.

Le système dit de sous-traitance globalement décrit, nous allons maintenant tenter d'analyser en quoi les deux grands problèmes de ces dernières années peuvent ou non modifier les principes fondamentaux de son fonctionnement. 


\section{La hausse du yen : un impact purement conjoncturel ?}

La forte hausse du yen a provoqué une évolution structurelle de la division nationale du travail, c'est-à-dire de la pyramide de sous-traitance. En effet, selon les secteurs et branches d'activité, la capacité concurrentielle des produits japonais par rapport aux produits étrangers s'est considérablement affaiblie, entraînant de la part des grandes entreprises donneuses d'ordres une accélération des stratégies de délocalisation à l'étranger, ou d'importation de pièces ou ensembles, auparavant commandés à des sous-traitants japonais.

\section{1. Évolution quantitative de la sous-traitance}

La traduction de ces stratégies peut se lire quantitativement à travers l'évolution de la part des PMI sous-traitantes sur le total des PMI, ou encore à travers l'évolution des quantités commandées aux entreprises concernées.

Le tableau 1 montre clairement la rupture qui s'opère dans les années récentes. En effet, alors que le nombre de PMI sous-traitantes rapporté au total des PMI était en hausse constante jusqu'à l'enquête nationale sur la soustraitance de 1981, celle de 1987 aboutit au résultat inverse.

La tendance est la même quel que soit le secteur de référence ; qu'il s'agisse des industries à faible recours à la sous-traitance (industries de l'agro-alimentaire) ou de celles qui en font un usage massif (matériel de transport), le différentiel avec 1981 est frappant ${ }^{16}$, la perte étant de plus de 5 points (tableau 2).

Cette rupture, pour importante qu'elle soit, ne doit cependant pas occulter le fait que toute évolution des stratégies d'approvisionnement de la part des grandes entreprises a un impact non négligeable sur les PMI japonaises, la plupart d'entre elles ne pouvant survivre en dehors du cadre du système de sous-traitance.

La hausse brutale du yen à l'automne 1985 a eu des effets directs et indirects sur les PMI sous-traitantes. Les effets directs se situent essentiellement sur le court terme, et constituent une réponse immédiate des grandes entreprises pour résorber la hausse du yen. Les effets indirects qui s'inscrivent dans une évolution à plus long terme sont, soit des répercussions des mesures d'adaptation immédiates, soit des retombées de la redéfinition des stratégies des donneurs d'ordres, notamment en matière d'internationalisation de la production.

16. L'enquête nationale sur la sous-traitance n'a lieu que tous les 6 ans (5 ans autrefois); les résultats fournis par le dernier livre blanc sur les PME font référence à l'enquête de décembre 1987. 
TABLEAU 1

Pourcentage de PMI sous-traitantes, industrie manufacturière

\begin{tabular}{ll}
\hline 1966 & 53,3 \\
1971 & 58,7 \\
1976 & 60,7 \\
1981 & 65,5 \\
1987 & 56,6 \\
\hline
\end{tabular}

N.B. : pourcentage de PMI sous-traitantes = (nombre de PMI sous-traitantes/nombre de PMI de l'industrie manufufacturière) $\times 100$

TABLEAU 2

Pourcentage de PMI sous-traitantes dans quelques branches industrielles

\begin{tabular}{llll}
\hline & 1976 & 1981 & 1987 \\
\hline Agro-alimentaire & 14,5 & 17,5 & 8,6 \\
Chimie & 37,1 & 38,5 & 22,9 \\
Mécanique générale & 82,7 & 84,2 & 75,0 \\
Matériel de transport & 86,2 & 87,7 & 81,2 \\
\hline
\end{tabular}

N.B. : pourcentage de PMI sous-traitantes = (nombre de PMI sous-traitantes/nombre de PMI de l'industrie manufacturière) x 100

Source: Chûshô kigyô hakusho (livre blanc sur les PME) 1976; 1980, p. 156; 1987, p. 141 ; 1989, p. 102.

La première réaction des grandes entreprises donneuses d'ordres, face à la hausse du yen, a été une compression des commandes en sous-traitance, que ce soit en volume ou en valeur. En fait, la tendance était déjà à la diminution des commandes enregistrées par les PMI sous-traitantes avant la hausse du yen, mais celle-ci s'est brusquement accélérée ensuite.

Le rapport des PMI sous-traitantes faisant état d'une augmentation des commandes en volume (par rapport à la même période de l'année précédente), à celles faisant état d'une diminution, qui passait de 16 à 13 au cours de l'année 1984 , devient négatif dès $1985\left(0,8\right.$ au $1^{\text {er }}$ trimestre, $-20,6$ au $\left.4^{\mathrm{e}}\right)$. L'année 1986 , marquée par la poursuite de la dégradation, laisse cependant envisager une reprise, confirmée en 1987 par un retour à un rapport positif dès le $3^{\mathrm{e}}$ trimestre.

En ce qui concerne l'évolution des commandes en valeur, la tendance était également à la baisse avant la hausse du yen, mais cela n'a rien de surprenant dans le contexte japonais où les PMI sous-traitantes doivent en permanence réaliser des gains de productivité qui se répercutent sur le prix des transactions selon le procédé analysé. Le rapport utilisé comme indicateur est donc toujours négatif, mais alors qu'il fluctue de -10 à -15 jusqu'en 1985, il 
descend brutalement dès le $4^{\mathrm{e}}$ trimestre $1985(-21,3)$ pour atteindre $-53,2$ au début de l'année 1987. La reprise s'amorce ensuite pour revenir aux normes antérieures à la fin de 1988.

Cette accélération de tendances antérieures est confirmée par l'enquête nationale sur la sous-traitance de décembre 1987 , où $83 \%$ des donneurs d'ordres ont reconnu avoir renforcé leur demande de baisse des prix des transactions, et où $27 \%$ d'entre eux ont pris pour mesure de rapatrier en interne des productions auparavant sous-traitées.

\subsection{Un phénomène conjoncturel induisant des changements structurels}

Si l'on regarde maintenant les raisons invoquées par les PMI pour expliquer la diminution des commandes reçues au cours de la période concernée, on remarque que c'est principalement la baisse du CA (chiffre d'affaire) du donneur d'ordres qui est à l'origine d'une telle situation, qu'il s'agisse d'une baisse du CA réalisé au Japon ou à l'exportation. La reprise en interne de production par le donneur d'ordres vient ensuite mais assez loin derrière. La délocalisation du donneur d'ordres à l'étranger, invoquée par seulement $14,5 \%$ des entreprises qui ont eu à faire face à une baisse des commandes entre 1985 et 1987, prend par contre du poids dans la période suivante $(26,8 \%)$, du moins pour la faible proportion de PMI qui continuent, malgré la reprise, à enregistrer une diminution des commandes (tableau 3).

Qu'il s'agisse des raisons de la baisse ou des raisons de la hausse après la reprise, l'impact de l'évolution du CA du donneur d'ordres montre bien l'aspect conjoncturel du phénomène. Cette constatation ne doit cependant pas masquer l'importance qu'il y a lieu d'accorder à des raisons, certes minoritairement invoquées, mais qui signifient en fait l'émergence de tendances plus lourdes pour le long terme. Les effets directs, soit l'impact accrue de la délocalisation du donneur d'ordres et l'accroissement de l'importation de pièces étrangères, constituent d'abord une réponse conjoncturelle, mais deviennent, dans un deuxième temps, des facteurs structurels qui induisent à leur tour des effets indirects, eux aussi porteurs de restructuration, tels la diversification vers de nouveaux donneurs d'ordres, la concentration des commandes vers un nombre plus limité de sous-traitants, ou encore le pourcentage de PMI qui ont reçu des commandes pour de nouvelles pièces qu'elles ne fabriquaient pas auparavant (tableau 3).

Bien que le pourcentage d'entreprises concernées par chacun de ces éléments soit souvent faible, il devient en fait plus significatif par le potentiel qu'il détient en termes de restructuration/mutation du système, lorsque replacé dans le contexte japonais du fonctionnement de la sous-traitance. 
TABLEAU 3

Évolution des quantités commandées en sous-traitance : raisons invoquées (pourcentage d'entreprises)

\begin{tabular}{lrr}
\hline & $1985-1987$ & $\mathbf{1 9 8 7 - 1 9 8 8}$ \\
\hline Total échantillon & 100,0 & 100,0 \\
PMI ayant enregistré une hausse des commandes & 32,8 & 39,2 \\
PMI ayant eu des commandes stables & 22,2 & 53,5 \\
PMI ayant enregistré une baisse des commandes & 45,0 & 7,3 \\
Raisons de la baisse, total échantillon & 45,0 & 7,3 \\
baisse CA du donneur d'ordres au Japon & 56,8 & 42,3 \\
baisse CA du donneur d'ordres à l'exportation & 56,5 & 47,2 \\
délocalisation du donneur d'ordres & 14,5 & 26,8 \\
importation pièces étrangères par donneur d'ordres & 3,3 & 5,6 \\
donneur d'ordres a changé de fournisseur & 9,2 & 16,9 \\
reprise en interne par le donneur d'ordres & 18,7 & 16,2 \\
diverses autres raisons & 8,6 & 11,3 \\
Raison de la hausse, total échantillon & 32,8 & 39,2 \\
hausse CA du donneur d'ordres au Japon & & 70,9 \\
hausse CA du donneur d'ordres à l'exportation & & 17,6 \\
augmentation nombre donneurs d'ordres & & 32,5 \\
concentration des commandes par le donneur d'ordres & & 13,3 \\
hausse de la sous-traitance par le donneur d'ordres & & 8,3 \\
commandes de nouvelles pièces ou processus & & 27,6 \\
arrêt importation de pièces étrangères par le donneur d'ordres (DD) & & 0,9 \\
diverses autres raisons & & 1,1 \\
\hline N.B. : en raison des reponses mo & & \\
\hline
\end{tabular}

N.B. : en raison des réponses multiples, le total est supérieur à 100.

Source : Chûshô kigyô hakusho (livre blanc sur les PME) 1989, p. 104. Enquête de décembre 1988.

C'est donc à travers l'analyse de ces éléments que nous allons tenter de décrire les évolutions prévisibles de la sous-traitance dans les années à venir. En effet, si la hausse du yen a été relativement vite digérée par les entreprises japonaises sans bouleversement structurel majeur, du moins apparemment, c'est parce que les mécanismes de régulation qu'offre le système de sous-traitance ont fonctionné pleinement. Ces mécanismes permettent une réaction immédiate sur le court terme, mais entraînent nécessairement une restructuration à plus long terme. En bref, le système a la capacité d'absorber les dysfonctionnements conjoncturels parce qu'il impulse sa propre restructuration. Un retour à une situation identique à la phase d'avant dysfonctionnement est inconcevable. C'est du moins ce qui ressort de l'analyse sur une longue période des diverses 
récessions qu'a connu l'économie japonaise. Certes, le système de sous-traitance ne peut-être considéré comme le seul élément régulateur de l'économie japonaise, mais son rôle est particulièrement important dans ce domaine ${ }^{17}$.

La récession liée à la hausse du yen n'échappe donc pas à la règle. Toutefois, force est de constater que l'impact qu'elle a eu au regard de l'internationalisation de l'économie japonaise est de nature à déstabiliser le système plus qu'aucune autre récession par le passé. Bien qu'elle n'ait fait qu'accélérer un processus déjà en cours, il n'en demeure pas moins vrai que les PMI japonaises se sont brusquement trouvées confrontées à un problème dont elles n'avaient pas encore pleinement pris conscience.

\section{Délocalisation et politiques d'approvisionnement}

\subsection{La délocalisation à l'étranger des grandes entreprises donneuses d'ordres}

Les grandes entreprises donneuses d'ordres qui ont ouvert une ou plusieurs unités de production à l'étranger n'ont pas encore, dans la grande majorité des cas, substitué de fournisseurs locaux à leurs fournisseurs japonais. En 1987, près de $80 \%$ d'entre elles déclaraient en effet, faire venir des pièces et sousensembles produits au Japon. Mais si, cette même année, seules $23,5 \%$ de ces entreprises avaient un taux d'achats locaux de plus de $70 \%$, contre $35 \%$ un taux inférieur à $5 \%$, leurs prévisions à trois ans étaient inverses; $35 \%$ devant passer à plus de $70 \%$ contre $15 \%$ restant en dessous de $5 \%$. Il ne fait aucun doute que l'impact de la délocalisation à l'étranger des donneurs d'ordres va continuer à se renforcer dans les années à venir. La hausse brutale du yen a en quelque sorte été, pour les sous-traitants, le détonateur d'une prise de conscience d'un risque pourtant tendanciellement bien réel, même si l'évolution se faisait en douceur, mais qu'ils avaient, semble-t-il, largement sous-estimé ${ }^{18}$.

17. Pour plus de détail sur ce point, voir Yveline Lecler (1981).

18. Lors d'enquêtes de terrain effectuées en septembre 1985 auprès de fournisseurs/ sous-traitants de l'automobile, secteur pionnier dans la délocalisation, notamment aux USA, cette absence d'inquiétude quant à l'impact éventuel d'un tel mouvement a pu être relevée. Ils y voyaient plutôt un moyen d'accroître leur production, le donneur d'ordres leur commandant les pièces nécessaires à leur production sur le sol américain. En 1987, certaines de ces mêmes entreprises, à nouveau visitées, connaissaient des difficultés liées à la hausse du yen et se montraient beaucoup moins positives par rapport aux activités outre-mer. Quelques-unes avaient d'ailleurs déjà décidé ou envisagé de suivre le donneur d'ordres dans son aventure aux États-Unis. 
À la fin de 1988, près de $28 \%$ des entreprises de l'industrie manufacturière disposaient au moins d'une usine à l'étranger (dont 6,6\%, plus de 3), tandis qu'un $10 \%$ supplémentaire projetait une prochaine implantation outremer. Bien que selon $66 \%$ des entreprises donneuses d'ordres, la production à l'étranger ne devrait pas avoir d'impact sur les quantités produites au Japon, on est en droit de se demander comment des investissements productifs de substitution à l'exportation pourraient rester, du moins à terme, sans effet sur ce point. Certes, le gouvernement japonais s'est lancé dans une politique de relance par la demande intérieure, mais celle-ci ne garantit pas totalement l'augmentation des commandes auprès des sous-traitants japonais qui se trouvent, également du fait de la hausse du yen, confrontés à une intensification de la concurrence des pays asiatiques, et voient poindre, marginalement encore, l'importation de pièces en provenance de ceux-ci.

\subsection{L'Importation de pièces étrangères}

Les donneurs d'ordres japonais, lorsqu'ils ne délocalisent pas à l'étranger notamment, ont en effet tendance à rechercher une baisse des coûts par cette voie. Comparativement peu invoquée pour expliquer la baisse des commandes enregistrée par certaines PMI sous-traitantes, il suffit de se référer au pourcentage de donneurs d'ordres qui, à la fin de 1988 avouaient avoir substitué des importations de pièces ou composants produits à l'étranger à des commandes à leurs sous-traitants japonais, pour considérer que les PMI sous-traitantes minimisent ce facteur. Ceci est d'ailleurs illustré par l'évolution de la sphère dans laquelle les donneurs d'ordres recrutent aujourd'hui, et surtout pensent le faire à l'avenir, par rapport au passé récent, c'est-à-dire avant la hausse du yen. Alors que $84 \%$ d'entre eux choisissaient principalement leurs fournisseurs/sous-traitants à l'intérieur de leur réseau d'entreprises affiliées (keiretsu kigyo) avant le hausse du yen, ce pourcentage tombe à $54,8 \%$ à la fin de l'année 1988 , et à $29,2 \%$ en perspectives d'avenir. Cette évolution se fait au profit d'une redistribution nationale plus large qui s'émancipe de la priorité à donner au réseau de soustraitance, notamment pour la période actuelle $(37,5 \%$ des firmes contre $14 \%$ avant hausse du yen). En ce qui concerne l'avenir, la sélection semble devoir se répartir à peu près également entre les entreprises intégrées au réseau, la totalité des firmes du territoire national et celles des pays étrangers. C'est le facteur " coûts » qui semble prévaloir dans le cas d'une sélection d'entreprises étrangères, les facteurs « qualité » associé aux « délais de livraisons » dans le choix des entreprises affiliées, et la recherche "d'une capacité technologique spécialisée » avec un bon rapport «qualité/coûts » et «délais de livraisons » qui favorise l'ensemble national, hors réseau intégré. 


\subsection{Les stratégies d'adaptation des sous-traitants}

Face à cette situation, et pour contrer la chute tendancielle de l'activité liée aussi bien à la délocalisation à l'étranger qu'à l'importation de pièces et composantes étrangères par les donneurs d'ordres, c'est principalement vers une tentative de diversification que se tournent les PMI sous-traitantes. Qu'il s'agisse de la pénétration d'une autre branche industrielle ( $46,3 \%$ des firmes concernées) ou de la recherche de nouveaux clients $(33 \%)$, cette diversification est d'abord envisagée dans le cadre de la sous-traitance.

Alors que le nombre moyen de donneurs d'ordres par sous-traitant était de 3 en 1976, il passe à 5 en 1987. La moyenne est toutefois peu significative tant les disparités sectorielles sont grandes. Ainsi dans le textile, l'évolution sur ce point est minime avec un passage de 2 à 3 , tandis que dans les produits dérivés du métal, il est de 5 à 10, et dans l'impression/imprimerie, de 6 à 12 .

Par contre, un pourcentage quasi équivalent ( $42 \%$ ) des PMI sous-traitantes compte renforcer ses relations avec un ou quelques donneurs d'ordres particuliers. Ce qui peut paraître contradictoire ne l'est en fait nullement dans le contexte japonais. Dans le cas, par exemple, d'entreprises sous-traitantes très intégrées à un donneur d'ordres précis, la recherche de nouveaux clients pour compenser les pertes liées à la diminution des commandes du donneur d'ordres principal n'est pas antagoniste avec un renforcement de l'intégration à ce donneur d'ordres. Du point de vue du sous-traitant, la stabilité acquise de longue date grâce aux commandes de ce donneur d'ordres, la maîtrise technologique et l'élévation des compétences obtenues grâce à son assistance sont autant de mérites qui valent bien quelques sacrifices, en termes de baisse des coûts et de redéploiement technico-industriel, par exemple. Du point de vue du donneur d'ordres, que le sous-traitant soit capable et prêt à faire les efforts nécessaires pour s'adapter à la situation, c'est-à-dire essentiellement qu'il puisse prendre en charge des ensembles plus complets que par le passé ou assurer conjointement davantage de fonctions, fait encore souvent pencher la balance en faveur d'une poursuite des relations établies de longue date. De plus, les avantages liés à la connaissance mutuelle équivalent à une quasi-culture d'entreprise commune, par rapport au coût d'une négociation avec un nouveau partenaire (qui présente de toute façon un risque) et deviennent un atout supplémentaire dans ces relations. Ces avantages seront-ils suffisants à l'avenir, face à un différentiel coût/ qualité qui s'accroît en défaveur des sous-traitants japonais (les pays d'Asie améliorant nettement la qualité de leurs productions), et face à l'exigence des pays d'accueil occidentaux quant à un taux d'achats locaux en hausse ?

Plus de $30 \%$ des PMI sous-traitantes envisagent par ailleurs de développer des produits propres ou du moins de commercialiser leurs produits par leurs soins. Ce chiffre, bien qu'en augmentation par rapport au passé, ne doit pas être 
interprété comme une volonté massive des sous-traitants de se désengager totalement de la sous-traitance. La sécurité qu'apporte celle-ci reste encore fortement présente, comme nous venons de le mentionner. Ce qui est recherché par ce biais est davantage une substitution partielle et progressive d'une partie des transactions de sous-traitance par mise sur le marché d'un, puis de plusieurs produits. Bref, il s'agit d'une compensation des pertes enchaînées par la baisse des commandes ou d'un moyen d'accroître le CA, alors que l'augmentation des transactions devient de plus en plus aléatoire. Cette dynamique peut certes déboucher à terme sur une prise d'indépendance vis-à-vis de la sous-traitance, et les pouvoirs publics ont récemment pris des dispositions pour aider les PMI dans cette voie, mais telle n'est pas encore la volonté affichée par la majorité des PMI sous-traitantes qui se tournent vers le développement de produits propres.

\section{Conclusion}

Bien que la hausse du yen, qui paraissait devoir rester une constante de la nouvelle donne mondiale, se soit estompée, les phénomènes qu'elle a engendré ou, plus exactement, dont elle a accrue le déploiement, notamment la délocalisation à l'étranger, ne semblent plus pouvoir être enrayés. Sans que l'on puisse parler de remise en cause fondamentale du système d'approvisionnement des grandes entreprises nippones, il semble bien que soient en train d'émerger des modalités de gestion des relations clients/fournisseurs qui tout en s'appuyant sur les principes antérieurs posent néanmoins les jalons d'une redéfinition des rôles respectifs au sein du " groupe ». Pour être en mesure de tenir ces nouveaux rôles, ou pour se préparer à une sortie du " groupe ", les PMI sous-traitantes œuvrent dans plusieurs directions. Que la volonté première soit une diversification par les produits ou processus, ou par la multiplication des donneurs d'ordres, il leur faut être en mesure de produire des ensembles de plus en plus complexes. Ceci implique l'acquisition de compétences nouvelles et secondairement l'investissement dans des équipements nouveaux. En ce qui concerne les compétences considérées comme nouvellement indispensables pour survivre en tant que sous-traitant de premier rang, on relève principalement les capacités en recherche et développement, les capacités à effectuer des essais, à faire de l'assistance technique, à négocier les prix des transactions. Des actions sont également jugées nécessaires pour améliorer certains points faibles tels que les compétences en conception de nouveaux produits, les capacités gestionnaires, surtout dans le domaine des délais de livraisons.

Tout ceci fait référence au renforcement de la volonté des donneurs d'ordres de ne plus avoir pour sous-traitants directs que des entreprises auxquelles 
ils peuvent déléguer une partie des charges qu'ils assuraient autrefois. Certes, la tendance au passage de la commande de pièces unitaires à des sous-ensembles complets, voire à des produits finis, était déjà visible avant la hausse du yen et l'accélération de l'internationalisation, mais c'est plus de la moitié des firmes qui se voient aujourd'hui confier ce type de production. De plus, le pourcentage de PMI sous-traitantes à qui il est demandé de concevoir le sous-ensemble en question est également en hausse pour atteindre un tiers des cas. Soutenir la concurrence des nouveaux pays industrialisés implique en effet de se redéployer vers des prestations supérieures. Les PMI japonaises sont amenées à abandonner à ces pays les pièces ou composants relativement simples pour se tourner vers ceux qui ont une plus forte valeur ajoutée, de remonter la filière vers la conception et le développement, et de la descendre vers l'assemblage. Celles qui ne seront pas capables de suivre dans cette voie seront reléguées au rang de soustraitant de deuxième niveau, chargées de fournir les pièces à celles qui y seront parvenues, tant que ces dernières ne seront pas contraintes elles aussi de s'approvisionner auprès d'entreprises de nouveaux pays industriels.

Dans l'immédiat, les grandes entreprises donneuses d'ordres réduisent leurs prestations d'assistance technique et de conseil, mais entendent que ce soit le sous-traitant de premier rang qui prenne le relais pour ses propres fournisseurs/sous-traitants, rôle qu'il joue certes déjà de par le fonctionnement même du système, mais qu'il devra dorénavant assumer à un niveau accru de complexité. Il doit en effet maîtriser, assister, définir la politique d'approvisionnement pour un nombre plus grand de pièces ou de processus en recherchant les coûts les plus bas possibles, sans dégradation de la qualité et des délais de livraisons, et ce, non plus sur un segment de la production, mais sur toute l'étendue de la filière, plus large, qu'il couvre. En résumé, il se substitue au donneur d'ordres pour l'ensemble fini qu'il produit et doit donc assumer pleinement la responsabilité qui va de pair.

C'est ce repositionnement des sous-traitants qui explique les besoins en compétences nouvelles qu'affichent les PMI sous-traitantes. Si elles veulent rester fournisseur de premier rang, il leur faut devenir petit à petit de véritables partenaires ${ }^{19}$ de leur donneur d'ordres, c'est-à-dire rompre avec la logique de la

19. Le terme de partenaire, en transcription phonétique du mot anglais, est nouvellement utilisé au Japon pour qualifier un type de coopération entre donneurs d'ordres et fournisseur de premier rang, semble-t-il, lorsque la relation comporte une dimension recherche et développement. Le sens du terme ne recouperait donc pas exactement le concept de partenariat qui se développe dans les pays occidentaux, et qui, bien qu'il n'y ait pas encore de définition communément admise, s'applique à la création de relations plus égalitaires, basées sur le long terme, etc. Dans ce sens, c'est l'expression japonaise «entreprise coopérante» (kyoryoku kigyô) qu'il conviendrait plutôt d'employer. Sur ces questions et notamment le problème de définition, voir Lecler (1991) 
quasi-intégration ${ }^{20}$, comme l'ont fait les entreprises, en général de relativement grande taille, qui ont d'ores et déjà pu passer le cap.

Cette évolution de la répartition des responsabilités entre donneurs d'ordres au sommet de la pyramide et fournisseurs de premier rang laisse supposer une relation plus égalitaire que par le passé, en ce qui a trait au pouvoir, entre les deux échelons, d'où la notion de partenaire plutôt que de quasi-atelier. Ceci n'implique pas nécessairement un affaiblissement de la dépendance au regard du chiffre d'affaires, ni d'ailleurs une diminution de la coopération. Les relations passées ont en effet créé un climat de confiance qui semble de nature à permettre une telle évolution sans remise en cause fondamentale du système. Et ce d'autant plus que les niveaux inférieurs, entreprises de taille plus réduite, restent quant à eux dans la logique de la quasi-intégration, non seulement par leur dépendance quant à leur chiffre d'affaires, mais aussi relativement aux transferts de technologies et de compétences. Ces évolutions « hiérarchisées » constituent, selon nous, un élément supplémentaire au maintien d'un système qui, malgré les évolutions en cours, semble devoir encore garder, pour un temps du moins, ses spécificités.

\section{Bibliographie}

De Banville J. et L. Chanaron (1990), dans M. Jacot (éd.) Du Fordisme au Toyotisme? Etudes et recherches du Commissariat Général du Plan, nos 2-3, La Documentation Française, février, p. 107.

ENRIETTI, A. (1990), "Les rapports de pouvoir et de collaboration : Filière, quasiintégration verticale et réseaux ", Cahiers de Recherche du GREPME 90-15, $18 \mathrm{p}$.

LECLER, Y. (1981), «Les petites et moyennes entreprises japonaises et la régulation de l'activité des grandes entreprises ", Revue d'Économie Industrielle, $\mathrm{n}^{\circ} 17,4^{\mathrm{e}}$ trimestre, p. 42-60.

LECLER, Y. (1991), «Relations partenariales et transfert de compétences : Le Japon, modèle de référence? ? Rapport au PPSH Rhône-Alpes, janvier, $111 \mathrm{p}$.

SATo Y. (1983), "Changement de la situation et orientation du système de soustraitance » (jyôkyô henka to shitauke shisutemu no dôkô) dans Structure de soustraitance de l' industrie automobile (jidôsha kôgyô ni okeru shitauke keiretsu kôzô), Shôkô kinyû, vol. 33, nos 5-6, juin, p 18.

TREVOR, M. et C. IAN (1988), « Manufacturers and suppliers in Britain and Japan; competitiveness and growth of small firms », PSI Policy Studies Institute, Londres, $144 \mathrm{p}$.

20. Sur la quasi-intégration, voir notamment l'excellente synthèse et analyse de Enrietti (1990). 
Ueda, H., W. ANasz et K. Yamamoto (1987), «Industrial structures in Japan », partie 1 «Pyramidal organization in the automobile and the electrical/electronic industries » et partie 2 « The pricing of procured parts and services in the automobile and the electrical/electronic industries ». University of Tokyo, Institute of Social Science, Occasional papers in Labor Problem and Social Policy, $\mathrm{n}^{\circ} 1$, février et $\mathrm{n}^{\circ} 2$, décembre. 